\title{
The Status of the University of Michigan Polarized Proton Target*
}

\author{
R.S. Raymond ${ }^{1}$, D.G. Crabb ${ }^{2}$, V.V. Fimushkin ${ }^{3}$, A.D. Krisch ${ }^{1}$, \\ A.M.T. Lin ${ }^{1}$, V.G. Luppov ${ }^{1}$, C.C. Peters ${ }^{1}$, A.I. Mysnik ${ }^{4}$, A.F. Prukoglyad ${ }^{4}$, \\ and $\mathrm{K}$. Yonehara ${ }^{1}$ \\ ${ }^{1}$ Randall Lab of Physics, University of Michigan, Ann Arbor MI 48109-1120, U.S.A. \\ ${ }^{2}$ Department of Physics, University of Virginia, Charlottesville VA 22901, U.S.A. \\ ${ }^{3}$ JINR, Dubna, Russia \\ ${ }^{4}$ IHEP, Protvino, Russia
}

\begin{abstract}
The University of Michigan Solid Polarized Proton Target (PPT) was built in the late 1980 's; it uses irradiated $\mathrm{NH}_{3}$ at $5 \mathrm{~T}$ and $1 \mathrm{~K}$. It was used in a 1990 experiment in a $24 \mathrm{GeV}$ intense proton beam at the Brookhaven AGS, where its average polarization was about $85 \%$. It was recently upgraded for the $70 \mathrm{GeV}$ SPIN@U-70 experiment at IHEP-Protvino in Russia. Improvements were made to its superconducting magnet, its refrigerator, and its NMR and microwave systems.
\end{abstract}

The University of Michigan Solid Polarized Proton Target (PPT) [1], shown in Fig. 1, is a $5 \mathrm{~T}$ and $1 \mathrm{~K}$ target using frozen ammonia $\left(\mathrm{NH}_{3}\right)$ as the target material. It was built in the late 1980's and was very successfully used in a p-p elastic scattering experiment [2] at the Brookhaven AGS in 1990. The target thickness is about $2 \times 10^{23}$ polarized protons $/ \mathrm{cm}^{2}$. The extracted AGS beam intensity of $10^{11}$ protons $/ \mathrm{s}$ on target was limited by quenches in the PPT magnet; hence, the polarized proton luminosity was about $2 \times 10^{34} / \mathrm{cm}^{2}$ s, with frozen, pre-irradiated ammonia as the target material. The PPT's proton polarization averaged about $85 \%$ during the 3-month experiment. Many subsystems were upgraded for use in the SPIN@U-70 p-p elastic scattering experiment [3] at the $70 \mathrm{GeV}$ U-70 accelerator at IHEP-Protvino in Russia.

The PPT magnet consists of a set of superconducting coils which produce a vertical $5 \mathrm{~T}$ field with a uniformity of better than $10^{-4}$ inside the $32 \mathrm{~mm}$-long material holder. The magnet's original main power supply failed; thus we purchased a new American Magnetics uni-polar power supply with an energy absorber; this system was successfully tested and then used with the PPT magnet.

\footnotetext{
*Supported by a U.S. Department of Energy Research Grant.
} 
The $213 \mathrm{MHz}$ NMR system, used to measure the proton polarization, was upgraded with a new PC using LabView with National Instruments boards. A voltage ramp to an FM signal generator provides a frequency ramp into two Liverpool NMR Boxes. Signals from the Boxes are processed by a home-built manual amplifier/offset module and then fed back to the PC for analysis. Two new 8-mm-diameter NMR coils are both perpendicular to the beam axis, but are centered at different radii to monitor polarization non-uniformity caused by the high intensity beam. These coils are small and equal in size to avoid some problems encountered in earlier NMR polarization measurements at $213 \mathrm{MHz}$.

The PPT target is cooled by a ${ }^{4} \mathrm{He}$ evaporation refrigerator. A new calibrated RuO resistor from Scientific Instruments was installed and tested; it agrees with temperature measurements from ${ }^{4} \mathrm{He}$ vapor-pressure to within about $1 \%$ at $1.5 \mathrm{~K}$. A new holder for the ammonia target material was made of Kel-F, with aluminum beam windows. The holder design allows fast replacement, with no soldering or unsoldering of the NMR coils. The $70 \mathrm{GeV}$ extracted proton beam at the U-70 accelerator may be initially rastered only vertically; thus, a rectangular material holder, with inside dimensions of $5 \mathrm{~mm}$ wide by $20 \mathrm{~mm}$ high by $32 \mathrm{~mm}$ long, was also fabricated and successfully tested.

For the earlier AGS experiment, the helium pumping system consisted of three Roots blowers in series $\left(6000 \mathrm{~m}^{3} / \mathrm{hr}\right.$ followed by $3000 \mathrm{~m}^{3} / \mathrm{hr}$ followed by $\left.350 \mathrm{~m} / \mathrm{hr}\right)$, backed by a $60 \mathrm{~m}^{3} / \mathrm{hr}$ mechanical pump. For SPIN@U-70, we would use two 5400 Roots blowers in parallel, backed by three $227 \mathrm{~m}^{3} / \mathrm{hr}$ mechanical pumps in parallel. These blowers and mechanical pumps were purchased and successfully tested. A pump system stand was fabricated, and remotely-controlled valves were purchased.

A system of rails and linear bearings was designed to allow easy movement of the PPT, either to remove it from the beam path or adjust its position slightly, for the different $\mathrm{P}_{\perp}{ }^{2}$ settings of the experiment's 35-meter-long Recoil Proton Spectrometer. We plan to use a 3001 liquid helium buffer dewar inside the radiation shielding enclosure. We recently purchased a 6301 helium dewar to bring liquid helium from the IHEP liquifier; the helium would be transferred from the 6301 dewar to the buffer dewar using a long transfer line through the 2-meter-thick shielding roof.

To avoid air transportation difficulties, we may irradiate the frozen ammonia at an electron accelerator at Moscow State University. The $140 \mathrm{GHz}$ microwaves would be produced with the Varian EIO tube used at the AGS, which has an output of about $22 \mathrm{~W}$. A more modern Varian power supply for the tube was obtained to replace the 35-year old supply used in the AGS experiment.

The schedule for SPIN@U-70 is now uncertain, because after a brief test run, SPIN@U-70 as delayed due to Russian customs difficulties. 


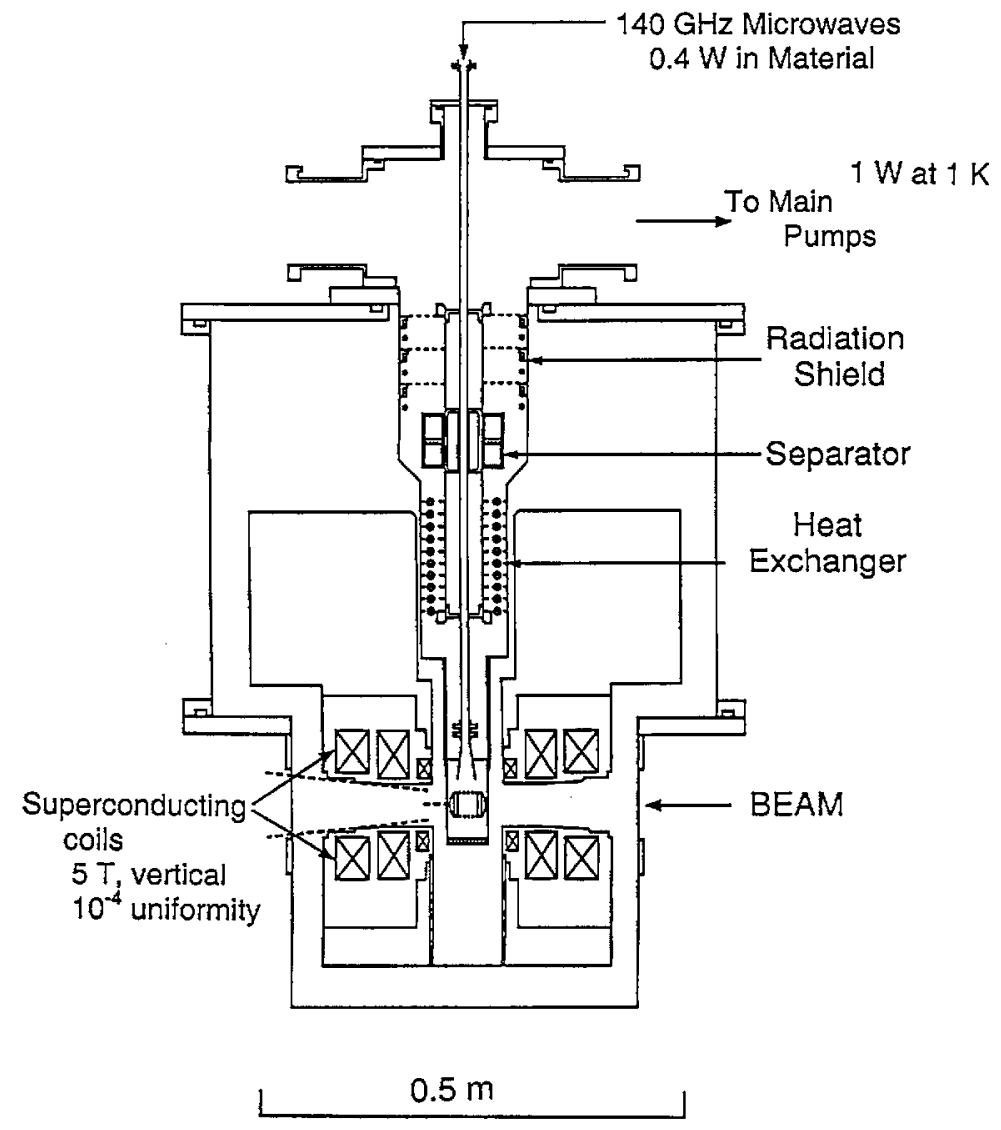

Fig 1. The Michigan Solid Polarized Proton Target

\section{REFERENCES}

1. D.G. Crabb, C.B. Higley, A.D. Krisch, R.S. Raymond, T. Roser, J.A. Stewart, G.R.Court, Observation of a 96\% Proton Polarization in Irradiated Ammonia, Phys. Rev. Lett. 64, 2627 (1990).

2. D.G. Crabb et al., High-Precision Measurement of the Analyzing Power in Large- $\mathrm{P}_{\perp}{ }^{2}$ Spin-Polarized $24 \mathrm{GeV} / \mathrm{c}$ Proton-proton Elastic Scattering, Phys. Rev. Lett. 65, 3241 (1990).

3. For SPIN@U-70 Proposal and details, refer to the Michigan Spin Physics Center website: http://spinbud.physics.lsa.umich.edu. 